\title{
Coopérer avec les parents
}

Une préoccupation des professeurs des écoles stagiaires?

Is cooperation with parents a concern of primary school trainee teachers? Mit den Eltern zusammenarbeiten. Eine Sorge der Grundschulreferendare? Cooperar con los padres. Una preocupación de los maestros en prácticas?

\section{Pascale Garnier}

\section{(2) OpenEdition}

\section{Journals}

Édition électronique

URL : http://journals.openedition.org/rechercheformation/1754

DOI : 10.4000/rechercheformation. 1754

ISSN : 1968-3936

Éditeur

ENS Éditions

\section{Édition imprimée}

Date de publication : 1 mars 2012

Pagination : 121-134

ISBN : 978-2-84788-374-9

ISSN : 0988-1824

\section{Référence électronique}

Pascale Garnier, « Coopérer avec les parents », Recherche et formation [En ligne], 69 | 2012, mis en ligne le 01 mars 2014, consulté le 30 avril 2019. URL : http://journals.openedition.org/ rechercheformation/1754; DOI : 10.4000/rechercheformation.1754 


\title{
Coopérer avec les parents
}

\section{Une préoccupation des professeurs des écoles stagiaires?}

\author{
$>$ Pascale GARNIER \\ Université Paris 13, UFR LSHS, laboratoire EXPERICE (Centre de recherche \\ interuniversitaire expérience ressources culturelles éducation, EA 2358)
}

RÉSUMÉ - En France, savoir coopérer avec les parents est devenu une compétence professionnelle institutionnellement attendue des professeurs des écoles. Nous analysons comment cette injonction trouve un écho dans l'expérience des enseignants débutants grâce à l'investigation d'un corpus d'écrits réflexifs produits à la fin de l'année de stage. Nous montrons en particulier que la focalisation sur le travail dans la classe avec les élèves, peut faire obstacle à une prise en compte des rapports avec les parents. Ceuxci s'inscrivent en miroir des préoccupations des stagiaires centrées sur la gestion de la classe et des apprentissages. Le contexte des stages dans les écoles, renforce la tendance à privilégier les seules relations individuelles et un rapport utilitariste à la coopération.

MOTS-CLÉS • école primaire, enseignant stagiaire, coopération, parents, formation initiale des enseignants

$\mathrm{Si}$ " le système scolaire français ne possède pas de culture de la coopération avec les parents " (Kherroubi, 2008, p. 11), des transformations sont à l'œuvre qui, à travers une série de textes réglementaires, marquent la place et le rôle croissants des parents et des associations de parents d'élèves dans les établissements scolaires, et au-delà (MEN, 2006a). Du point de vue de la formation des enseignants, la coopération avec les parents fait également l'objet de nouvelles injonctions institutionnelles en tant que compétence professionnelle attendue des enseignants. Après la Suisse romande en 1992 (Perrenoud, 1996) et le Québec en 2001 (Larivée, 2010), la France indexe la formation des enseignants à un référentiel de compétences professionnelles intégrant la dimension des relations avec les parents. Le cahier des charges de la formation des maîtres publié en décembre 2006 (MEN, 2006b), présente en annexe un référentiel de dix compétences dont la neuvième est intitulée "Travailler en équipe et coopérer avec les parents et les partenaires de l'école ". Elle stipule notamment que le professeur est capable de " communiquer avec les parents, en contribuant à l'établissement d'un dialogue constructif dans le but de les informer sur les objectifs de son enseignement ou de son activité... ". La place des 
parents apparaît aussi dans l'énoncé de la première compétence de ce référentiel "Agir en fonctionnaire de l'État et de façon éthique et responsable ". Outre sa déclinaison pour l'école maternelle (MEN, 2009), les récentes transformations de la formation des enseignants ont conservé un référentiel de dix compétences que les professeurs doivent maîtriser pour l'exercice de leur métier (MEN, 2010). La spécificité de cette compétence qui porte sur les dimensions collectives du métier d'enseignant (Gather Thurler \& Perrenoud, 2005 ; Lessard et al., 2009), interroge tout particulièrement les débuts de son exercice et, au niveau de l'école primaire, la coopération avec les parents. La mise en place d'actions de formation dans ce domaine appelle en effet à développer des recherches auxquelles ce texte entend contribuer par une investigation centrée sur les professeurs des écoles stagiaires.

\section{Début dans le métier et collaboration avec les parents}

Là où d'autres pays ont commencé à investir ce domaine du partenariat avec les familles dans la formation des enseignants du premier degré (Guerdan, 2005 ; Larivée, 2008), il semble qu'en France les investigations sur les débuts dans le métier soient très largement centrées sur l'activité de l'enseignant dans sa classe. Les travaux de recherche insistent généralement sur une véritable épreuve que constitue l'entrée dans le métier pour les enseignants débutants : un " choc de la réalité ", qui peut être synonyme de douloureuse initiation, moment d'ébranlement ou de remaniement identitaire (Crahay et al., 2010). Outre l'analyse de l'appropriation des " règles " du métier (Méard \& Bruno, 2009), les travaux sur les débuts dans la profession font état de trois stades de préoccupations des stagiaires : mettre au travail, avancer dans le programme, vérifier si l'enseignement dispensé débouche bien sur les apprentissages visés (Maulini, 2009, p. 67). L'analyse des trajectoires de professeurs des écoles marque cette centration des problématiques professionnelles sur les rapports avec les élèves : la gestion de la classe et celle des apprentissages (Marquie-Dubie, 2009), mais aussi l'importance d'un travail d'équipe entre enseignants dans les écoles (Sembel et al., 2009 ; Hoff, 2009). Si l'entrée dans le métier peut représenter un " passage de l'autre coté du miroir " qui fait de la formation initiale un espace de tensions structurelles entre les statuts et les rôles de l'élève et de l'enseignant (Dubois, Gasparini \& Petit, 2006), ces préoccupations relatives aux parents d'élèves parviennent-elles à émerger face à une focalisation sur tout ce qui se joue d'abord avec les élèves, dans la classe ? Et si oui, comment, à quelles occasions, dans quel type de situation? Quelles représentations des rapports entre les enseignants et les parents véhiculent-elles?

Les travaux de recherche marquent la grande diversité des termes utilisés pour analyser les rapports entre les parents et les enseignants : partenariat, coopération, collaboration, participation, implication, coéducation... Au-delà d'un inévitable flou sémantique, ces termes se rapportent à un principe de symétrie et de réciprocité, comme celui de " communauté éducative " inscrite dans la loi d'orientation sur 
l'éducation de 1989. On sait que, depuis cette époque, les critiques n'ont pas cessé de dénoncer le fossé entre ce principe et la réalité, ses illusions aussi quand c'est fondamentalement l'école qui détermine son " ouverture " aux familles et qu'elle occulte leur forte diversité socioculturelle. Ces critiques sont récurrentes et bien documentées (Glasman, 1992 ; Dubet, 1997 ; Périer, 2005 ; Duru-Bellat \& Van Zanten, 2006). Si l'on s'attache au point de vue des acteurs, la question fondamentale que pose tout travail collectif est d'abord celle de leur propre exigence de coordination de l'action. Loin d'être présupposée, elle doit être analysée par la recherche, en même temps qu'il est nécessaire d'interroger comment le travail collectif met à l'épreuve l'activité propre de l'enseignant et son identité professionnelle (Garnier, 2005). De ce point de vue, une série d'investigations empiriques dans des écoles primaires montre l'importance des contextes locaux d'enseignement, la priorité donnée à une individualisation et une personnalisation des relations entre parents et enseignants, une complexification de la collaboration avec les familles qui s'inscrit dans des dynamiques d'équipes pédagogiques très contrastées (Garnier, Kherroubi $\&$ Monceau, 2008). Peut-on dire que ces priorités sont celles des professeurs des écoles stagiaires? Comment la complexité et l'ambivalence des relations entre les enseignants et les parents prennent-elles du sens dans l'expérience des stagiaires?

\section{Méthode : corpus et analyse}

Pour étudier la manière dont les professeurs des écoles stagiaires intègrent (ou non) la dimension d'un travail collectif avec les parents dans leur découverte de l'exercice du métier, nous avons utilisé un matériel d'enquête particulier : des "écrits réflexifs ", réalisés par les stagiaires à la fin de leur année de stage, dans l'académie de Créteil. D'une autre manière que le "mémoire professionnel " auquel il a pour ainsi dire succédé, l'écrit réflexif se veut un outil au service de la dimension réflexive de l'activité enseignante, auquel participe au premier chef sa dimension d'écrit (Crinon, 2003 ; Gonin-Bolo \& Benoit, 2004). En même temps, cet écrit constitue le support du " bilan personnel de compétences " réalisé à l'issue de l'année de stage, inscrit dans le cahier des charges de la formation des maîtres de 2006. Dans cet écrit, si les stagiaires ne sont pas obligés de traiter systématiquement des dix compétences professionnelles du référentiel, il leur est demandé de faire état des compétences qu'ils pensent avoir acquises, ou non, en relation avec leur formation et les stages effectués en responsabilité dans les classes, soit trois stages dans chacun des trois cycles du primaire : un stage filé sur l'ensemble de l'année scolaire et deux stages groupés de trois semaines. Ainsi, tout en étant incités à le faire, les stagiaires ont l'initiative de parler, ou non, des relations avec les parents, selon la manière dont ils se représentent cet écrit et cette année de stagiaire.

L'intérêt méthodologique d'utiliser ces écrits comme matériel d'enquête est double. D'une part, contrairement à une enquête par questionnaire qui soumet les réponses aux seules catégories de questions qui sont celles du chercheur, 
l'investigation des écrits réflexifs permet de prendre la mesure des préoccupations qui sont celles des stagiaires ${ }^{1}$. Elle permet de contextualiser ces préoccupations des stagiaires en les mettant en rapport avec leur propre pratique. D'autre part, contrairement à des entretiens individuels ou collectifs, l'investigation de ce matériel permet de limiter les effets d'imposition de cette thématique qui conduirait à surestimer son importance dans l'ensemble des préoccupations des professeurs stagiaires. En effet, toute technique d'enquête directe ne peut que présupposer que cette coopération avec les parents est importante ; elle introduit un rapport aux valeurs, qui n'est pas nécessairement celui mis en jeu par les stagiaires. Même s'il ne permet pas de rendre compte de leurs pratiques effectives, ce corpus permet en outre de neutraliser les effets de biais relatifs au volontariat des enquêtés.

Un corpus de 167 écrits réflexifs de professeurs des écoles stagiaires, de 3 à 8 pages, a été recueilli en juin 2009 et juin 2010, c'est-à-dire les deux dernières années précédant la mise en place des masters " enseignement " pour le premier degré, auprès d'une dizaine de formateurs de trois centres départementaux, de manière à relativiser leur forme d'accompagnement. L'anonymat des conditions de recueil par les formateurs interdit de mettre en relation son analyse avec des variables relatives aux stagiaires (propriétés sociales, cursus et niveaux d'études, etc.) et aux contextes socio-scolaires des trois lieux de stage. Leur formation dans ce domaine de la collaboration avec les familles, ne fait pas l'objet d'un enseignement dédié, mais d'interventions le plus souvent aléatoires et ponctuelles.

Dans un premier temps, nous avons repéré systématiquement toutes les occurrences des termes " parent(s) ", " père(s) " " mère(s) " " famille(s) ", ainsi que les adjectifs et adverbes qui leur sont liés, afin de comptabiliser les écrits selon leur nombre d'occurrences. Dans un second temps, nous avons réalisé une analyse thématique de contenu en construisant un codage de ces occurrences selon leur signification et leur contexte d'énonciation, de manière à en indiquer un ordre de fréquence selon leur distribution dans les écrits. Suivant une analyse inductive, qualitative et comparative des données (Glaser \& Strauss, 2010), la construction des catégories de codage est réalisée à partir de ces occurrences, plutôt que déterminée a priori par une typologie générale des domaines, des formes ou des degrés de coopération entre les enseignants et les parents. Le codage permet de distribuer ces occurrences en cinq catégories : l'énoncé institutionnel de la compétence, l'intégration des parents dans la dimension collective du travail enseignant ; la position particulière de stagiaire vis-à-vis des parents ; les outils et les objets de communication avec les parents; la référence à des élèves particuliers qui ont suscité des relations avec leurs parents. II permet ainsi de prendre la mesure

1 C'est par exemple le cas du sondage réalisé par l'institut CSA (2010) auprès de 1150 professeurs des écoles de moins de 5 ans d'expérience qui leur demande d'évaluer la " place des parents à l'école " : plutôt satisfaisante (59\%) ; trop importante $(21 \%)$; pas assez importante $(19 \%)$; ne sait pas (1\%). 
des manières dont cette dimension du travail collectif participe, ou non, de la découverte des réalités du métier par les professeurs des écoles stagiaires.

\section{Résultats}

\subsection{Un métier avec des élèves ... et des adultes}

La question de la coopération avec les parents n'est pas une des préoccupations majeures des professeurs des écoles stagiaires qui se donnent à lire dans leurs écrits réflexifs. En effet, près de $40 \%$ des écrits ne contiennent aucune occurrence des termes " parent(s) ", " famille(s) ", " mère(s) ", " père(s) ", ou leur déclinaison lexicale ; $47 \%$ des écrits mentionnent une, deux ou trois occurrences et seuls $13 \%$ des écrits marquent quatre occurrences et plus (jusqu'à 18 occurrences). La place des parents dans ces écrits est à l'image de leur rareté dans les rapports de visite de stage et de leur absence dans la mention d'un formateur ou d'une formation dans ce domaine. La seule mention explicite de la neuvième compétence (14 occurrences) est le plus souvent formelle, réduite à son énoncé ; seul un écrit s'attache à décliner ses différentes dimensions. Bien que les écrits réflexifs soient en principe indexés à ce référentiel, celui-ci est rarement investi de manière systématique et encore moins exploité par les stagiaires pour analyser ce qu'ils peuvent dire du travail avec les parents. Dans ces écrits, l'usage du terme " parent(s) " (225 occurrences) l'emporte largement sur les termes "famille(s) " et "familial " (29 et 16 occurrences). Le terme " parents " qui signifie des individus et une catégorie d'acteurs, a supplanté les termes "famille " et "familial " qui renvoyaient implicitement aux classes populaires et, plus largement à l'idée d'un " milieu " socialement différencié (Glasman, 1992). Les affectations successives des stagiaires dans les écoles peuvent donner matière à contraster fortement les conditions socio-scolaires d'exercice du métier et contribuer à renforcer les effets d'évidence d'un sociologisme spontané, où se télescopent un classement en zone d'éducation prioritaire, un quartier, un milieu familial et social et le comportement des élèves en classe ${ }^{2}$. Aussi, les termes " famille " et " familial " renvoient souvent à la question de "l'hétérogénéité " des élèves et à une autre compétence professionnelle du référentiel : " prendre en compte la diversité des élèves ".

Pour une trentaine d'écrits, les parents apparaissent parmi l'ensemble des acteurs qui font du métier d'enseignant un travail collectif avec des adultes, voire plus largement dans l'ensemble des "paramètres " avec lesquelles il compose nécessairement : "Je ne savais pas qu'un PE doit prendre en compte autant de

2 Notons qu'un seul écrit fait usage d'une référence savante dans ce domaine des relations avec les familles, en l'occurrence le nom emblématique de Bourdieu, et implicitement à la thèse de La reproduction publiée en 1970 avec Passeron. Son schématisme et son évidence contribuent sans doute à un volontarisme qui consiste à en prendre le contrepied. Seuls trois écrits font référence aux différences culturelles et ethniques entre parents, alors même que, dans ce domaine, le recrutement des professeurs des écoles dans l'académie s'est dans une certaine mesure diversifié (Charles, 2006). 
paramètres : les projets d'école, les disponibilités des lieux, la disponibilité du matériel, les parents, les collègues, les femmes de service... Je pensais que le professeur était le seul maître à bord. ", écrit une stagiaire. Les parents représentent comme une nouvelle donne, qu'il faut intégrer dans la représentation de l'exercice d'un " métier solitaire ", ou que les stages imposent comme une réalité à prendre effectivement en compte : "Je n'y avais pas vraiment réfléchi ". La dimension collective du métier apparaît comme ce qui déborde de la classe et y prend nécessairement sa source : "Néanmoins je pense que la gestion de classe ne se passe pas uniquement en classe. Elle s'étend au sein de l'école et concerne l'ensemble de l'équipe pédagogique, ainsi que la famille. " Si la gestion de la classe est sans doute le premier motif pour donner droit de cité aux parents, la question des apprentissages des élèves appelle aussi l'implication des parents, la manière d'en " tirer parti " dans la mesure où " Elle prolonge le travail fait en classe et peut ainsi influer largement sur la réussite scolaire des enfants."

C'est donc le travail avec les élèves qui entraîne un travail en dehors de la classe avec leurs parents, dans le but de faciliter ce travail en classe. Seuls deux écrits font état d'un risque de voir les parents " empiéter " sur les prérogatives des enseignants, occupant symboliquement le centre de la classe. Si les écrits marquent généralement l'importance des échanges avec les parents, voire de leur écoute, affaire essentiellement de psychologie interindividuelle, un seul fait état d'une forte exigence de réciprocité et utilise le terme de " coéducation". Cet écrit est aussi le seul à présenter la relation école-famille comme " une relation historiquement compliquée mais qui se veut complémentaire et non subsidiaire "; son auteur, qui se présente comme ancienne salariée d'une Fédération de parents d'élèves, n'illustre pas sa place dans son expérience de stagiaire. Cet écrit est d'ailleurs le seul à faire état de l'importance des associations de parents d'élèves.

\subsection{Face aux parents : stagiaire ou " enseignant à part entière " ?}

Selon qu'il est filé ou groupé, le stage en responsabilité donne lieu à des relations différentes avec les parents marquées dans six écrits. En effet, le stage groupé ne manque pas de faciliter aux yeux des stagiaires leur installation dans l'école, leur disponibilité, la possibilité d'être un " référent " ou " l'interlocutrice privilégiée des parents ", par rapport au titulaire. Plus largement, vingt-cinq écrits font état de cette différence entre l'état de stagiaire et celui d'un " enseignant à part entière ". La médiation du titulaire de la classe paraît ici décisive, notamment en stage filé quand il s'agit, par exemple, d'être " la maitresse du mardi " et non pas une étudiante, une décharge de direction ou une remplaçante. En tout cas, figurer comme " enseignant à part entière " aux yeux des parents, c'est l'être en même temps auprès des élèves ; double construction en miroir où l'un ne va pas sans l'autre : "En effet, l'importance qu'attribuent les élèves au statut de l'enseignant dépend de la valeur que lui attribuent les parents. "Si pour des étudiants, une future identité professionnelle en tant qu'enseignant se construit en opposition à la figure du parent (Mackiewicz, 
2010), notre corpus montre qu'elle se joue en même temps dans la classe, " contre " la double figure de l'élève et du stagiaire.

Tout un lexique est mobilisé pour qualifier la nature de cette différence entre le stagiaire et l'enseignant : fonction, statut, position, place, posture. Ces termes indiquent une différence à la fois abstraite, symbolique, et incarnée, matérielle ; deux registres qu'il s'agit précisément de faire coïncider dans une même " personnalité professionnelle". Ils supposent des transactions à la fois intra et intersubjectives où la représentation de soi-même compose avec le regard des parents et réciproquement. De ce double investissement à la fois institutionnel et subjectif dépendent aux yeux des stagiaires la confiance et l'autorité, la crédibilité et la responsabilité, la légitimité qu'ils mettent en jeu face aux parents. Être un " enseignant à part entière " consiste avant tout à se présenter en tant que " professionnel " vis-à-vis des parents, définis inversement comme non professionnels. Ce registre de l'expertise institue une hiérarchie avec le parent, qui impose au stagiaire d'être à la hauteur de cette exigence.

Avec l'idée que "l'école est une institution, pas une famille ", une dizaine d'écrits soulignent la "distance à instaurer " entre l'enfant, ses parents et l'enseignant, celui-ci devant le plus souvent "s'interdire des relations maternelles avec les enfants". Dans un contexte où les stagiaires du primaire sont souvent des jeunes femmes sans enfant, et où les parents sont la plupart du temps des mères, ce terme est trois fois plus présent dans les écrits que celui de père. Ils s'interrogent expressément sur la dimension " maternelle " des relations avec les élèves, notamment en petite section: " Je ne me sentais pas dans la peau d'une enseignante, mais plutôt dans celle d'une nourrice ou d'une assistante maternelle ". Il s'agit alors d'" éviter le maternage et la régression ", tout en prenant la mesure de l'importance d'une " sécurité affective " des tout petits. La question de la discipline et des règles impersonnelles d'une vie scolaire apparaît ici centrale aux yeux des stagiaires pour se distancier d'un registre de l'affectivité, de la familiarité et du domestique qui caractérise les parents. Elle ressort d'une expertise en tant que " professionnel ", mais aussi d'une autorité déléguée à l'enseignant en tant que " représentant de l'État dans son école".

\subsection{Outils et objets de communication avec les parents}

Seize écrits relèvent des outils de communication avec les parents, ou font apparaître l'objet de ces communications. Outre les réunions collectives dans la classe, figurent d'abord les " mots" dans le bien nommé " carnet de correspondance ". Le cahier de texte, où sont notés les devoirs à faire à la maison, est mentionné une seule fois. À travers ces objets et les élèves eux-mêmes, se donnent à voir une présence à distance des parents dans la classe, le sentiment d'être " jugé par les parents " et, réciproquement, la place qu'occupe l'école au sein des familles. Les stages peuvent être ainsi l'occasion d'une " prise de conscience ", 
de " déclics " qui, au lieu de frontières bien tranchées entre espaces scolaire et domestique, en donnent à percevoir des contaminations, des chassés croisés, ou encore des sas que matérialise la question du respect des horaires d'entrée et de sortie des élèves.

Les sanctions et les punitions, règlement ou règles "à faire signer aux parents ", prennent la première place des objets de communication dans ces écrits, s'agissant de s'assurer d'une double prise disciplinaire sur l'enfant-élève. Mais la question est posée : faire appel aux parents, est-ce un acte d'autorité ou un aveu d'impuissance? Vis-à-vis des élèves ou des parents ? Relative à des difficultés dans la classe, l'intervention auprès des parents revêt bien des significations ambivalentes : signe négatif d'une défaillance du stagiaire, "impression de faiblesse, d'incompétence" et/ou signe positif d'une incitation à leur " implication dans la vie scolaire de leur enfant ". L'évaluation et la notation du travail des élèves constituent le deuxième objet de communication avec les parents qui est relevé dans ces écrits, parfois lié à la compétence du référentiel qui lui est spécifique. À la question d'une motivation de l'élève à travers les notes qu'il rapporte à ses parents s'ajoute celle, plus fréquente, de la lisibilité, de la compréhension de l'évaluation par les parents et de la complexité de l'acte : " pour qui évaluer? "À l'école maternelle, au lieu du " cahier de vie ", l'usage du seul " livret de compétences " réduit la communication avec les parents aux " performances " des élèves. Enfin, un autre objet est cité de manière ponctuelle : "l'argent des parents " qu'il s'agit de récolter pour une sortie.

Au total, à l'exception des réunions avec les parents de la classe, rien n'est dit d'éventuels dispositifs collectifs de collaboration entre l'école et les familles, d'une participation des parents à la vie de l'école et, plus largement, à une vie sociale qui se tisse à travers l'école, témoignant là aussi d'une situation de stage qui renforce la place donnée aux relations individuelles avec les parents. Les différences de relations avec les parents à l'école maternelle et à l'école élémentaire sont cependant marquées dans une quinzaine d'écrits. Induits par les temps d'accueil quotidiens, des " échanges fréquents " et des " contacts directs " avec les parents caractérisent la maternelle, à l'image des liens avec des "enfants [qui] ne sont pas encore des élèves ", en petite section notamment. C'est surtout à ce niveau que les stagiaires peuvent souligner qu'il faut répondre à leur besoin partagé d'être rassurés et mis en confiance : " être là pour les enfants, c'est aussi être là pour les parents."

\subsection{Des relations au "cas par cas"}

Quinze écrits mettent en exergue un ou des " cas " d'élève(s) précis qui suscitent un travail particulier du professeur stagiaire. Sans exception, les difficultés des élèves sont ici au cœur des relations qui se tissent avec les familles : pour les comprendre et/ou y remédier en intervenant auprès de leurs parents. Parmi les difficultés dont les stagiaires font état, celles proprement scolaires sont très minoritaires (deux occurrences en cours préparatoire) ; elles soulignent des défaillances parentales, 
soit pour éviter un redoublement, soit pour assurer la fréquentation scolaire. Prédominent les difficultés de l'élève liées à un comportement jugé perturbateur pour les stagiaires et celles d'ordre socio-affectif qui, à une exception près, concernent des enfants de petite section et sont imputées à une sortie du " giron familial ". Aux yeux des stagiaires, la compréhension de la situation familiale, en particulier si elle passe par un entretien avec un parent, permet de " relativiser", voire de pacifier la situation. Ces difficultés peuvent en outre mobiliser le titulaire, plus rarement le directeur d'école, et de manière exceptionnelle les collègues et des spécialistes du RASED ou extérieurs à l'école. Dans le cas des comportements d'élèves jugés perturbateurs, elles donnent aussi aux stagiaires à mesurer leur relative "impuissance", d'autant qu'ils sont pour ainsi dire de passage dans l'école et que les difficultés en question apparaissent bien répertoriées sans pour autant faire l'objet d'intervention des titulaires.

Très rares sont les situations explicitement conflictuelles ; il est d'ailleurs signalé qu'elles demandent " assurance et maturité " qui peuvent faire défaut au " jeune âge " du stagiaire. Ainsi, une altercation entre une stagiaire et un père (un cas en maternelle située en ZEP), demande tout particulièrement de ne pas " se laisser envahir par ses émotions", " prendre du recul ", " avoir confiance en soi "; soit en un mot, "rester professionnelle ". De ces épreuves marquantes témoigne aussi le récit d'une rencontre avec la mère d'un élève ; pour en souligner le caractère dramatique, la stagiaire en rapporte une phrase placée sur la page de couverture de son écrit réflexif : "II ne me reste plus qu'à le tuer et à me jeter ensuite par la fenêtre ". Pourtant, après ce récit, il ne sera plus question de cette mère et de son fils dans les six pages suivantes de cet écrit. Ici aussi, l'essentiel reste ce qui se passe en classe, bon gré, mal gré, quand il faut "trouver des subterfuges " avec les élèves eux-mêmes. Soulignons que seul cet écrit met en cause une absence de " préparation " du stagiaire dans ce domaine.

\section{Conclusion}

Aucun écrit ne mentionne que " coopérer avec les parents " est une compétence que le stagiaire n'a pas acquise. Si les relations avec les parents s'avèrent parfois à " approfondir " ou, mieux, à " maîtriser ", c'est d'abord au fil des situations d'exercice du métier qu'elles semblent pouvoir l'être, de la même manière que c'est au cours du stage en responsabilité que les stagiaires ont pu les découvrir. Reste que son importance est toute relative au travail avec les élèves : placée au pourtour de la classe, elle répond avant tout aux situations qui en montrent la nécessité, en particulier la gestion des élèves jugés perturbateurs. Du même coup, à l'exception de la petite section, elle est étroitement focalisée sur des attentes scolaires, faisant rarement état d'autres préoccupations qui seraient relatives à la vie de l'enfant. Comme pour le travail collectif avec les collègues et des partenaires extérieurs à l'école (Garnier, 2005), il faut donc prendre la mesure des exigences 
variables de coordination de l'action avec les parents, qui ordonne l'existence même et les priorités du travail collectif à partir du travail de l'enseignant dans sa classe. Essentielle est donc précisément cette " structure cellulaire du travail enseignant " que représente la classe (Tardif \& Lessard, 1999) et qui constitue le cadre tangible de tout travail collectif entre adultes. Tout autant que le travail en équipe entre collègues, les relations des professeurs des écoles stagiaires avec les parents s'inscrivent dans un rapport utilitariste et sélectif à la collaboration : celle-ci est rarement posée comme une fin en soi, mais comme un moyen pour réaliser ce que l'enseignant s'attache à faire dans sa classe. Pour des stagiaires soucieux de se présenter comme des enseignants à part entière face aux parents, cette logique instrumentale peut toutefois apparaître $a$ priori contradictoire avec une autonomie professionnelle et les conditions des stages en responsabilité y faire effectivement obstacle, en premier lieu pour tout ce qui concerne une collaboration à l'échelle de l'école.

Sans doute, est-il nécessaire en formation initiale de permettre aux futurs enseignants de s'approprier des outils de compréhension des réalités et des enjeux de la coopération avec les familles qui dépassent les relations interpersonnelles. En outre, l'intégration d'un travail avec les familles dans la formation initiale, au titre par exemple des actions d'accompagnement scolaire, paraît propre à " approcher les réalités du métier d'élève et les contextes de vie des familles " (Francis \& Goï, 2011). Pour reprendre les termes de Bourdieu (2001), ces outils et ces dispositifs contribueraient à transformer une "réflexivité narcissique ", centrée sur leur singularité et leur expérience personnelle, en une "réflexivité réformiste ", c'est-à-dire à la fois collective et opératoire dans l'exercice du métier. Dans cette perspective, l'essentiel se joue d'abord dans les établissements scolaires, au niveau des dynamiques collectives et des dispositifs qui permettent pendant le stage, comme après la titularisation, de donner au débutant cette réassurance à partir de laquelle il peut s'autoriser à faire des parents de véritables partenaires, ou plus exactement à jouer de la pluralité des significations d'une coéducation (Garnier, 2010). Autrement dit, dans ce domaine tout particulièrement, l'essentiel ne serait pas de renforcer les impératifs pesant sur la formation initiale, mais bien de contribuer au développement d'une culture professionnelle collective. Le risque de réduire une compétence pratique en un objet de savoir (Rey, 2006), va ici de pair avec le risque de mettre la formation au seul service de la normativité des injonctions officielles et, paradoxalement, de renforcer l'individualisation des ressources que met en jeu la coopération avec les parents.

Pascale GARNIER

pascale.garnier@univ-paris13.fr 


\section{BIBLIOGRAPHIE}

BOURDIEU P. (2001). Science de la science et réflexivité : cours du collège de France, Paris : Raisons d'agir.

CHARLES F. (2006). "L'enseignement : une chance de mobilité sociale pour les jeunes issus des immigrations?", Formation-emploi, n 94, p. 59-72.

CRAHAY M., WANLIN P., ISSAIEVA E. et al. (2010). " Fonctions, structuration et évolution des croyances (et connaissances) des enseignants ", Revue française de pédagogie, $\mathrm{n}^{\circ} 172$, p. 85-129.

CRINON J. (dir.) (2003). Le mémoire professionnel des enseignants : observatoire des pratiques et levier pour la formation, Paris : L'Harmattan.

CSA (2010). Observatoire des professeurs des écoles débutants, $n^{\circ} 1000550$, Consultation pour le SNUIPP, Paris.

DUBET F. (dir.) (1997). École, familles : le malentendu, Paris : Textuel.

DUBOIS P., GASPARINI R. \& PETIT G., (2006). "Professeurs des écoles novices : formes collectives et individuelles du "passage à travers le miroir" ", Revue française de pédagogie, $\mathrm{n}^{\circ} 155$, p. 73-82.

DURU-BELLAT M. \& VAN ZANTEN A. (2006). Sociologie de l'école, Paris : Colin.

FRANCIS V. \& GOÏ C. (2011). " Intégrer et articuler le continuum d'expériences à la formation initiale des enseignants. L'exemple d'un programme de recherche sur l'accompagnement à la scolarité ", in L. Benchama \& D. Groux (dir.), Formation des enseignants et ouverture sur le monde : approches comparées, Paris : L'Harmattan. p. 89-115.

GARNIER P. (2005). "À l'école élémentaire : dynamique collective et recomposition du métier ", Recherche et formation, n 49, p. 35-50.

GARNIER P., KHERROUBI M. \& MONCEAU G., (2008). " Sur la coopération écolefamilles : les perspectives ouvertes", in M. Kherroubi (dir.), Des parents dans l'école, Ramonville Saint-Agne : Érès; Fondation de France, p. 206-216.

GARNIER P. (2010). " Co-éduquer à l'école maternelle : une pluralité de significations", in S. Rayna, M.-N. Rubio \& H. Scheu (dir.), Parents-professionnels : la coéducation en questions, Ramonville Saint-Agne : Érès, p. 119-126.

GATHER THURLER M. \& PERRENOUD P. (2005). " Coopération entre enseignants : la formation initiale doit-elle devancer les pratiques ? ", Recherche et formation, $\mathrm{n}^{\circ} 49$, p. 91-105.

GLASMAN D. (1992). " "Parents" ou "familles" : critique d'un vocabulaire générique ", Revue française de pédagogie, $\mathrm{n}^{\circ} 100, \mathrm{p} .19-33$.

GLASER B. G. \& STRAUSS, A. A. (2010). La découverte de la théorie ancrée : stratégies pour la recherche qualitative, Paris: Colin.

GONNIN-BOLO A. \& BENOIT J.-P., (dir.) (2004). Le mémoire professionnel en IUFM : bilan de recherches et questions vives, Saint-Fons : INRP. 
GUERDAN V. (2005). " La formation des enseignants au partenariat avec les familles: quel avenir ? " in E. Palacio-Quintin, J.-M. Bouchard \& B. Terrisse (dir.), Questions d'éducation familiale, Outremont : Ed. Logiques, p. 313-329.

HOFF C. (2009). " Parcours de formation et auto-construction des enseignants débutants du premier degré ", in R. Goigoux, L. Ria \& M.-C. Toczek-Capelle (dir.), Les parcours de formation des enseignants débutants, Clermont-Ferrand : Presses universitaires Blaise Pascal. p. 149-165.

KHERROUBI M. (dir.) (2008). Des parents dans l'école, Ramonville Saint-Agne : Érès ; Fondation de France.

LARIVEE S. (2008). "Collaborer avec les parents : portrait, enjeux et défis de la formation des enseignants au préscolaire et au primaire ", in M. E. Correa \& C. Gervais (dir.), Les stages en formation à l'enseignement : pratiques et perspectives théoriques, Québec: Presses de l'Université du Québec, p. 219-247.

LARIVEE S. (2010). " La collaboration école-famille au Québec ", in G. Brougère (dir.), Parents, pratiques et savoirs au préscolaire, Berne : P. Lang, p. 181-202.

LESSARD C. et al. (2009). " De quelques facteurs facilitant l'intensification de la collaboration au travail parmi les enseignants : le cas des enseignants canadiens", Éducation et sociétés, $\mathrm{n}^{\circ} 23$, p. 59-77.

MACKIEWICZ M.P. (2010). " Opposition à la figure du parent et identité enseignante ". Diversité, $\mathrm{n}^{\circ} 163$, p. 24-31.

MARQUIE-DUBIE H. (2009). "Trajectoire de professeurs des écoles : de l'année de préparation au concours à l'année de stagiarisation, la construction des problématiques professionnelles ", in R. Goigoux, L. Ria \& M.C. Toczek-Capelle (dir.), Les parcours de formation des enseignants débutants. Clermont-Ferrand : Presses universitaires Blaise-Pascal, p. 121-134.

MAULINI 0. (2009). "Devenir enseignant : invariants et évolution des débuts dans la profession ", in R. Goigoux, L. Ria \& M.C. Toczek-Capelle (dir.), Les parcours de formation des enseignants débutants, Clermont-Ferrand : Presses universitaires Blaise-Pascal, p. 61-78.

MEARD J. \& BRUNO F. (2009). Les règles du métier dans la formation des enseignants débutants, Toulouse : Octares.

MEN (2006a), La place et le rôle des parents dans l'école. Rapport conjoint IGEN et IGAEN, $\mathrm{n}^{\circ} 2006057$.

MEN (2006b). Cahier des charges de la formation des maîtres en IUFM, Arrêté du 19 décembre 2006, BO $\mathrm{n}^{\circ}$ 1, 4 janvier 2007.

MEN (2009). Déclinaison du référentiel de compétences des enseignants pour une formation des professeurs des écoles à l'exercice en école maternelle, Circulaire n² 2009-098, BO n 32 du 3 septembre 2009. 
MEN (2010). Définition des compétences à acquérir par les professeurs, documentalistes et conseillers principaux d'éducation pour l'exercice de leur métier, Arrêté du 12 mai 2010, B0 n 29 du 22 juillet 2010.

PERIER P. (2005). Ecole et familles populaires : sociologie d'un différend, Rennes : Presses universitaires de Rennes.

PERRENOUD P. (1999). Dix nouvelles compétences pour enseigner, Paris : ESF.

REY B. (2006). " Les compétences professionnelles et le curriculum : des réalités inconciliables? ", in Y. Lenoir \& M.H. Bouillier-Oudot (dir), Savoirs professionnels et curriculum de formation, Québec : Presses de l'université Laval, p. 83-108.

SEMBEL N., LEONARD F., TERUEL, B. et al. (2009). " L'entrée dans la carrière d'une cohorte de professeurs des écoles en Gironde. La construction de l'identité au travail : entre adaptation et autonomie ", in R. Goigoux, L. Ria \& M.C. ToczekCapelle (dir.), Les parcours de formation des enseignants débutants, ClermontFerrand : Presses universitaires Blaise-Pascal, p. 135-148.

TARDIF M. \& LESSARD C. (1999). Le travail enseignant au quotidien : expérience, interactions humaines et dilemmes professionnels, Bruxelles : De Boeck.

\section{Abstracts • Zusammenfassungen • Resúmenes}

\section{Is cooperation with parents a concern of primary school trainee teachers?}

ABSTRACT • In France, cooperation with parents has become a professional skill that is institutionally expected from primary school teachers. We examine how this injunction finds an echo in the experience of beginning teachers through the analysis of a corpus of reflective pieces of writing submitted in the late year of work experience. We argue that focusing on classroom work with pupils can put an obstacle to teacher-parents relations. They reflect the concerns of trainee teachers who prioritise classroom management and learning outcomes. Work experience in schools increases the trend towards focusing on individual relations only and on a utilitarian vision of cooperation.

KEYWORDS • primary school, trainee teachers, cooperation, parents, initial teacher education

\section{Mit den Eltern zusammenarbeiten Eine Sorge der Grundschulreferendare?}

ZUSAMMENFASSUNG • In Frankreich ist die Zusammenarbeit mit den Eltern eine berufliche Kompetenz geworden, die von Grundschullehrern institutionell erwartet wird. Wir analysieren, welchen Platz diese Vorschrift in der Erfahrung angehender Lehrer einnimmt, in dem wir ein Korpus reflexiver Schriften analysieren, die am Ende der Referendarzeit geschrieben wurden. Wir zeigen insbesondere, dass die Fokalisierung auf die Arbeit in der Klasse mit den Schülern ein Hindernis für die Beziehungen mit den Eltern darstellen kann, die sich in den Sorgen der Referendare über Klassenführung und Unterricht widerspiegelt. Der Kontext des Referendariats an der Schule verstärkt die Tendenz, nur die individuellen Beziehungen und eine utilitaristische Auffassung der Zusammenarbeit zu fördern.

STICHWÖRTER • Grundschule, Referendar, Zusammenarbeit, Eltern, Lehrergrundausbildung 


\section{Cooperar con los padres \\ Una preocupación de los maestros en prácticas?}

RESUMEN - En Francia, saber cooperar con los padres se ha convertido en una destreza profesional institucionalmente esperada por parte de los maestros. Analizamos cómo esta conminación encuentra resonancia en la experiencia de los docentes principiantes gracias a la investigación de un corpus de escritos reflexivos realizados al terminar el año de prácticas. Mostramos en particular que la focalización sobre el trabajo en el aula con los alumnos puede ser un obstáculo para la toma en cuenta de las relaciones con los padres. Estos reflejan las preocupaciones de los practicantes centradas sobre la gestión de la clase y de los aprendizajes. El contexto de las prácticas en la escuelas refuera la tendencia a privilegiar únicamente las relaciones individuales y una relación utilitarista a la cooperación.

PALABRAS CLAVES • colegio de educación primaria, profesor en prácticas, cooperación, padres, formación inicial del profesorado 